\title{
Optimization of Power Collector System for Large-scale Offshore Wind Farm Based on Topological Redundancy Assessment
}

\author{
Wei Shurong ${ }^{1}$, Feng Yuyao ${ }^{2}$, Liu Kunlun ${ }^{1}$ and Fu Yang ${ }^{1}$ \\ ${ }^{1}$ College of Electrical Engineering, Shanghai University of Electric Power, Shanghai 200090, China; \\ ${ }^{2}$ Electric power Research Institute of State Grid Shanghai Municipal Electric Power Company, Shanghai 200437, China
}

\begin{abstract}
Because of the bad environment of wind farms in the far -reaching sea, the cost of power collector system is high. The contradiction between economy and reliability of the power collector system planning is particularly prominent. According to the particularity of the wind farm in the far-reaching sea and the demand of the power collector system on higher reliability, this paper proposes the definition of topological redundancy of the power collector system and develops a multi-objective optimization model based on the topological redundancy. Thus, the contradictory variables of economy and reliability are optimized. Taking a large-scale offshore wind farm as an example, based on the topological redundancy assessment, the topology of its power collector system is optimized from the perspective of life cycle cost. The results show that, although the initial cost of the optimal redundancy topology is slightly higher than that of the radial structure, the advantage of life cycle cost after 8 years of operations is obvious, which can meet the actual engineering requirements of the power collector system for the wind farm in the farreaching sea.
\end{abstract}

\section{Introduction}

As the core of offshore wind farms, the power collector system is relevant to the operation efficiency, performance and economic benefits of offshore wind farms, and is the crucial equipment for safe and reliable operation of offshore wind farms [1-2]. With the largescale, clustered, and far-reaching Marine development of offshore wind power development [3-4], more uncertain factors in the ocean have put forward higher requirements for the reliability of the collector system, so it is urgent to put forward new optimization models and methods for the collector system that are more suitable for the development trend of far-reaching offshore wind power. Literature [5-6] establishes a basic model for the optimization of the power collector system, takes the investment cost of medium-voltage sea cable as the target, gives the optimal cost scheme meeting the ocean constraint conditions, and evaluates the reliability [6]. In literature [7-9], reliability is converted into economic cost for unified consideration. In literature [7-8], opportunity cost of failure is considered; in literature [9], network loss cost of medium-high voltage cable is considered. With the further advancement of the research, literature [10] gives the operation and maintenance cost model of the electricity collector system, and establishes the lifecycle cost (LCC) model of the offshore wind farm power collector system. From the overall perspective of
25 years' operation period, the sum of the offshore wind farm operation cost, maintenance cost and failure cost even exceed the construction cost. What's more, the collector system full life cycle costs include two parts: the economic cost and reliability cost. As a result, it's significant to take a comprehensive consideration of full life cycle cost of offshore wind farm power collector system.

At present, these models focus on radial structure [6] or ring structure [9] with mature engineering cases. Radial structure has lower cost but slightly lower reliability, while ring structure has higher reliability but higher cost. Literature [11-12] proposed that the reliability of the collector system could be improved through redundancy design, but the redundancy degree as well as the specific optimization model and method is not given. Offshore wind Farm power Collector system's large-scale, far-reaching cost up to hundreds of millions of dollars, and maintenance difficulties, make the contradiction between economy and reliability particularly prominent. As a result, It's urgent to seek a topological structure between radial structure and ring structure, which owns certain redundancy and optimize contradictory variables : economy and reliability, making the structure have certain cost advantages on the basis of higher reliability.

In this paper, the definition of the redundancy of the collector system is presented, and the optimal design of the redundancy of the collector system is sought through the optimization of the topology redundancy 
evaluation of the power collector system. Combined with a planned large-scale offshore wind farm case, the case results are analyzed from the comprehensive consideration of the full life cycle cost and reliability cost of the collector system, utilizing economic cost change curve of the typical topology structure of the power collector system and its advantages or disadvantages so as to provide data basis for the planning of far-reaching large-scale offshore wind farm.

\section{Redundancy of power collector system}

The power collector system of large-scale offshore wind farm mainly includes fans, submarine cables connecting each fan and offshore substations. Moreover, the power collector system runs through the wind power generation and contains numerous electrical equipment. Because of the wide variety of electrical equipment in the collector system and the variety of topological connection modes of the pressure cable, the investment cost and reliability of different connection schemes are greatly different, so there exists much space for optimization.

Due to the changeable connection mode and various equipment of offshore wind farm power collector system, the redundancy of the power collector system can be divided into equipment redundancy and structural redundancy. Taking the limited space of offshore fan engine room into consideration, the equipment redundancy is generally considered only low quality and consumable equipment. Therefore, this paper mainly focuses on the structural redundancy of the power collector system.

The structural redundancy of the power collector system of offshore wind farm is coupling with the number of fan series, the number of fans in each series and the topological form of fans in each series, considering the redundancy of connection mode based on commonly used topological structures in the power collector system like radial structure and ring structure.

Figure.1 shows several possible topologies for collector system with certain redundancy.

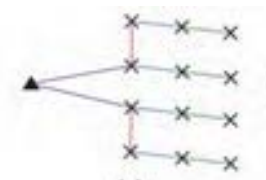

(a)

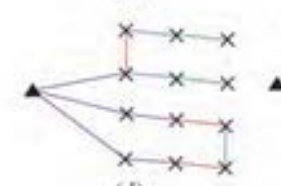

(d)

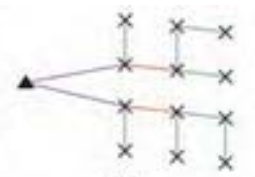

(b)

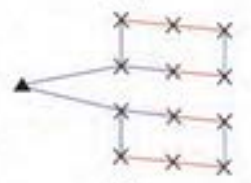

(c)

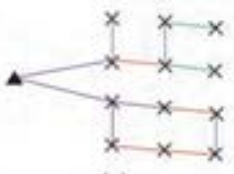

(c)

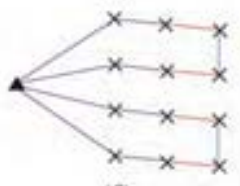

(f)
Fig. 1. Several topologies of power collector system.

Figure.1 (a) and (b) are radial topology, which are composed of a certain number of fans in series. The number of fans in a series is determined by the rated capacity of the fans and the quantity and rated load flow of submarine cable. Due to the simple structure and low investment cost, it is widely used. However, if one of the cables fails, fans behind will not operate normally and its reliability is poor. The topology structure with high reliability of the power collector system, as shown in Figure. 1 (e) and (f), is to connect a certain number of fans through sea cable to form a ring, and fan connection realizes all redundancy. Compared with the radial structure, the ring structure not only adds a section of cable, when one of marine cables becomes fault, remaining topological structure can still satisfy the requirements for all the fans to connect to the power grid through other normal marine cables, which greatly improves the reliability of the system. The number of large-section cables is also relatively large, and the cost increases correspondingly, resulting in higher initial investment cost. Considering the contradiction between economy and reliability of radial topology structure and bilateral ring structure of the power collector system, a partial ring structure of the collector system with economy and reliability between radial topology structure and bilateral ring structure is presented, as shown in Figure. 1 (c) and (d).

The partial ring structure is adopted in the power collector system, which makes the topology structure of the collector system meet certain reliability and provides the optimization foundation for better economy. However, for the partial ring structure of the power collector system, the different degree of redundancy affects the economy and reliability of the system. In order to study the economy and reliability of the optimal structure of the collector system with different redundancy degrees, the concept of redundancy degree is put forward to represent the redundancy degree of the power collector system.

Redundancy is a representation of degree of the topology redundancy based on the topology redundancy structure of the power collector system. The definition of redundancy gamma is as follows.

$$
\gamma=\frac{\sum_{z=1}^{g(i)} \frac{\sum_{v=1}^{f(i)} \boldsymbol{n}_{v}}{\boldsymbol{n}_{z}}}{n}
$$

In the formula: $\mathrm{n}_{\mathrm{z}}$ is the number of all possible paths of the fan in the area of the power collector system; $n_{V}$ is the number of paths in the topological series of the power collector system in each series.

$\mathrm{F}(\mathrm{i})$ is a nonlinear function representing the topological string number of the power collector system, which is a discrete variable. Given the optimization space of the topological series of the power collector system, its variation range is as follows.

$$
\frac{W I}{I_{S_{\max }}}<n<\frac{W I}{I_{S_{\min }}}
$$

In formula: $\mathrm{W}$ is the total number of fans in the area of the power collector system; $I_{S \max }$ and $I_{S \min }$ are the maximum and minimum section current carrying capacity respectively. I is the rated current of the wind farm fan in normal operation, and its expression is shown in formula (3). 


$$
I=\frac{S_{g}}{\varepsilon U_{m}}
$$

In formula: $\mathrm{S}_{\mathrm{g}}$ is the rated capacity of the fan; Is the conversion coefficient, which is $1.732 . \mathrm{U}_{\mathrm{m}}$ is the rated voltage of medium-voltage sea cable.

In the same way, the nonlinear function $\mathrm{g}(\mathrm{i})$ of the number of zones in the collector system is calculated according to the constraint of the high voltage sea cable flow. Among them, the selection of sea cable section should satisfy the requirements of long-term allowable submarine cable current and thermal stability check during short circuit, so as to make the selection of each submarine cable section feasible and optimal.

$$
I_{t, \max } \leq K_{t} I_{t, o}
$$

In formula, $\mathrm{I}_{\mathrm{t}, \mathrm{Max}}$ is the maximum continuous load current flow through a certain section of submarine cable. $\mathrm{I}_{\mathrm{t}, \mathrm{O}}$ is the long-term Marine cable current, provided by the Marine cable manufacturer; $\mathrm{K}_{\mathrm{t}}$ is the total correction factor of the long-term allowable Marine cable current. When the submarine cable is short circuited, the cross section of the thermal stability check cable is:

$$
S_{t, \text { min }}=\frac{I_{t, \infty} \sqrt{t_{l}}}{C_{t}}
$$

In formula: $S_{t, m i n}$ is the minimum cross section required for short circuit thermal stability of sea cable; $I_{t, \infty}$ is the steady short circuit current of the sea cable; $t_{1}$ is short circuit time of sea cable; $C_{t}$ is the thermal stability coefficient of sea cable.

\section{Multi-objective optimization model based on the redundancy of the power collector system}

The redundancy design can improve the reliability of the power collector system of far-reaching offshore wind farm, and the economy of the collector system should be taken into consideration in the redundancy design, so the redundancy design of the power collector system should ensure the reliability of the power collector system and make the economy better. Therefore, based on the redundancy of the power collector system, this paper establishes a multiobjective optimization model for offshore wind farm collector system.

From the perspective of the construction and operation life cycle of the offshore wind farm, the total cost of the collector system in the 25-year life cycle of the offshore wind farm includes initial investment cost, fault maintenance cost in later operation, power failure loss, network loss and salvage value recovery and disposal cost.

\subsection{Initial investment cost}

The difference of initial investment cost of diverse redundancy design schemes of the collector system mainly lies in the length, specification and number of medium voltage switchgear.

The initial investment cost of the collector system is shown in formula (6) .

$$
C_{1}(\gamma)=C_{C B}(\gamma)+C_{S W}(\gamma)
$$

$C_{C B}(\gamma)=\sum_{S=1}^{N_{S}} \sum_{f=1}^{N_{S f}} \sum_{c=1}^{N_{s f c}}\left(C_{\text {cable,sfc }}(\gamma)+C_{\text {install }}(\gamma)+C_{\text {trans }}(\gamma)\right)$

In formula: $\mathrm{C} 1$ is the initial investment cost; $\mathrm{C}_{\mathrm{CB}}$ is the cost of medium-voltage cable [9]; $\mathrm{N}_{\mathrm{s}}, \mathrm{N}_{\mathrm{sf}}$ and $\mathrm{N}_{\mathrm{sfc}}$ are respectively the number of offshore substations in large offshore wind farms, the number of feeders connected to the s offshore substation (the number of fan series), and the number of sea cables in the $f$ feeder of the $\mathrm{S}$ substation. $\mathrm{C}_{\text {cable }}$ and $\mathrm{SFC}$ are corresponding cable costs (including cable length and cable section), as shown in Equation (8); Cinstall and Ctrans are respectively the installation and transportation costs of Marine cables. Among them, medium-voltage cable laying is affected by the complex Marine environment, such as the uncrossing of submarine cables and laying in the same trench, etc., which are considered as constraint conditions in the model. In addition, in the practical application of the project, according to the results of sea sweeping survey, the influence of other factors such as the avoidance zone of sea cable laying will be considered, which will be optimized by setting a certain weight for the length of sea cable in the optimization solving algorithm [9].

$$
C_{\text {cable }, s f c}(\gamma)=L_{s f c}(\gamma) C_{\text {unit }}(\theta)
$$

In formula: $\mathrm{L}_{\mathrm{sfc}}$ is the length of the sea cable in section $\mathrm{C}$ of the Feeder in Section $\mathrm{F}$ in substation $\mathrm{S}$; $\mathrm{C}_{\text {unit }}\left({ }^{\theta}\right)$ for the segment of the submarine cable unit price, is generally proportional to the cable sectional area theta, through the load current, the maximum allowable voltage drops $\Delta \mathrm{V}_{\max }$ (associated with the length of each section of the submarine cable), maximum short-circuit current, etc. $\mathrm{C}_{\mathrm{SW}}$ is the cost of switching equipment, as shown in Equation (9).

$$
C_{S W}(\gamma)=C_{S A} N_{S A}(\gamma)
$$

In formula: $\mathrm{C}_{\mathrm{SA}}$ is the cost of each switching equipment; $\mathrm{N}_{\mathrm{SA}}$ is the number of switching devices.

\subsection{Failure maintenance cost}

During the entire operation cycle of offshore wind power system, after submarine cables or switching equipment fail due to navigation of offshore vessels, adverse environment and unknown uncertainties, afterservice maintenance is required to reduce the loss caused by shutdown due to failure. The operation and maintenance cost of fault maintenance [10] is.

$$
C_{M}(\gamma)=K_{a}(\gamma) C_{m, a}+K_{s}(\gamma) C_{m, s}
$$

In formula: $\mathrm{C}_{M}$ is the operation and maintenance $\operatorname{cost} ; \mathrm{K}_{\mathrm{a}}$ is the total number of feeder failures in one year; $\mathrm{C}_{\mathrm{m}, \mathrm{a}}$ is the cost required for single maintenance of the feeder; Ks is the total number of failures of feeder switch equipment in one year; $\mathrm{C}_{\mathrm{m}, \mathrm{s}}$ refers to the cost of single maintenance of feeder switch equipment. 


\subsection{Power failure loss and network loss}

Different redundancy design schemes of power loss collector system have different reliability. When submarine cable or switching equipment fails, some fans cannot work normally and are forced to stop, thus resulting in power generation loss of wind farm fans during fault maintenance, which is power failure loss. At the same time, the network loss of submarine cables is also related to the reliability of the collector system. In order to calculate the shutdown loss of the fan and the network loss of the sea cable, it is necessary to assume [7] : The failure probability of all locations of the submarine cable is equal; (2) The failure probability of switching equipment is equal; Due to the long average recovery time (Meantime to repair, MTTR), the average power loss of the fan during the failure period is equal to the average power of its annual normal power generation.

$$
P_{\text {lost }}=\frac{P_{\text {rate }} T_{\text {equal }}}{T}
$$

In formula: $P_{\text {lost }}$ is the average power lost by the fan during shutdown; $P_{\text {rate }}$ is the rated power of the shutdown fan; $T_{\text {equal }}$ is the annual utilization hours of the fan, take $2600 \mathrm{~h} ; \mathrm{T}$ is $8760 \mathrm{~h}$ a year.

$$
E_{\text {lost }}(\gamma)=\lambda(\gamma) t_{\text {MTTR }} P_{\text {lost }}
$$

In formula: $\mathrm{t}_{\mathrm{MTTR}}$ is the average recovery time; The electricity lost by $E_{\text {lost }}$ for failure; Is the number of fan failures over the course of a year at a wind farm.

$$
\begin{aligned}
& E_{\text {lost }}(\gamma)=\frac{\lambda(\gamma) t_{\text {MTTR }} P_{\text {rate }} T_{\text {equal }}}{T} \\
& C_{F}(\gamma)={ }_{c} E_{\text {lost }}(\gamma)
\end{aligned}
$$

In formula, $\mathrm{C}_{\mathrm{F}}$ is the fan power failure loss; $\mathrm{C}$ is the offshore wind power grid price, set at 0.85 yuan $/(\mathrm{kW} \cdot \mathrm{h})$. The network loss of submarine cable varies with the change of topological structure of the collector system, which can be expressed as mathematical expected value. The one-year network loss expected value of the I series/loop feeder can be expressed as:

$$
C_{O, i}(\gamma)=\sum_{j=1}^{m}{ }_{c} T p_{j}(\gamma) P_{j}(\gamma)+{ }_{c} T p_{c}(\gamma) P_{c}(\gamma)
$$

In formula:j is the number of fan shutdown caused by feeder $i$ fault; $m$ is the total number of fans attached to feeder $\mathrm{i} ; \mathrm{P}_{\mathrm{j}}$ is the probability when feeder I has $\mathrm{J}$ fans out of service; $P_{j}$ is the active power loss of feeder I with $\mathrm{J}$ fans out of service; $\mathrm{P}_{\mathrm{c}}$ is the probability of feeder I when no fan is out of service; $P_{c}$ is the active power loss of feeder I when the fan is out of service.

\subsection{Salvage value recovery and disposal cost}

Marine cable wire and switchgear are composed of nonferrous metals, which have certain recovery value. But the recovery of such equipment requires ships and manual labor, and the recovery value is not high, so this part of the cost can be considered to offset each other after treatment [16]. In conclusion, considering that $\mathrm{C} 1$ is a one-time investment, other costs have time value and need to be discounted. Aiming at the lowest life cycle cost of the collector system of offshore wind farms [17-18] and considering its constraints, an optimization model of the full life cycle cost of the collector system based on redundancy was established as follows:

$$
\begin{aligned}
& \min C(\gamma)=C_{1}(\gamma)+\beta\left(C_{O}(\gamma)+C_{F}(\gamma)+C_{M}(\gamma)\right) \\
& \text { s.t }\left\{\begin{array}{c}
I_{s f c}<I_{\text {rated }}(\theta) \\
\left|\Delta V_{s f c}\right|<\Delta V_{\max } \\
F_{x} \bigcap_{x, y \in S ; x \neq y} F_{y}=\phi \\
F_{x} \cup F_{y}=S \\
\beta=\frac{(1+\tau)^{t}-1}{\tau(1+\tau)^{t}}
\end{array}\right.
\end{aligned}
$$

In formula, $\mathrm{C}$ is the life cycle cost of the collector system; $I_{\text {sfc }}$ and $\Delta V_{\text {sfc }}$ respectively the s substation article of $\mathrm{f}$ feeder in the $\mathrm{c}$ section of submarine cable on the flow of electrical current and the voltage drop of this submarine cable; $I_{\text {rated }}(\theta)$ is the rated current of the selected cable; $F_{x}$ is a set of fan nodes. As sea cables cannot be laid across, constraints require that there is no intersection between fan clusters, and all fans should be included in the fan clusters. Is the present value and conversion coefficient of the annual investment expense; $I_{s}$ the discount rate; $T$ is the full life cycle, and the life of offshore wind farm is $20 \sim 25$ years.

Aiming at the all-radial or all-ring connection modes commonly seen in current researches, this paper proposes some partial redundant topological structures that may appear in actual engineering situations, and defines redundancy variables to represent the degree of redundancy of topology. The full life cycle cost of the collector system is taken as the optimization goal, and the redundancy is taken as an important variable of global cycle optimization to participate in the process of topology optimization, which breaks through the problem that reliability is only taken as the evaluation result and does not participate in the optimization process in the existing research. In the optimization process, the initial redundancy is set as 0 (i.e. a single series radial structure), and then step by step. Gradually increase (considering some redundancy, the maximum for ring structure corresponds to the maximum redundancy), equivalent to radiate to the ring and between each redundancy topology enter in step cycle optimization comparison of link, after comparing the minor cycle in each nested retain full life cycle cost minimum, at the end of the global search to get the full life cycle cost of the global optimal solution, output optimization results. The specific optimization flow chart is shown in Figure 2. 


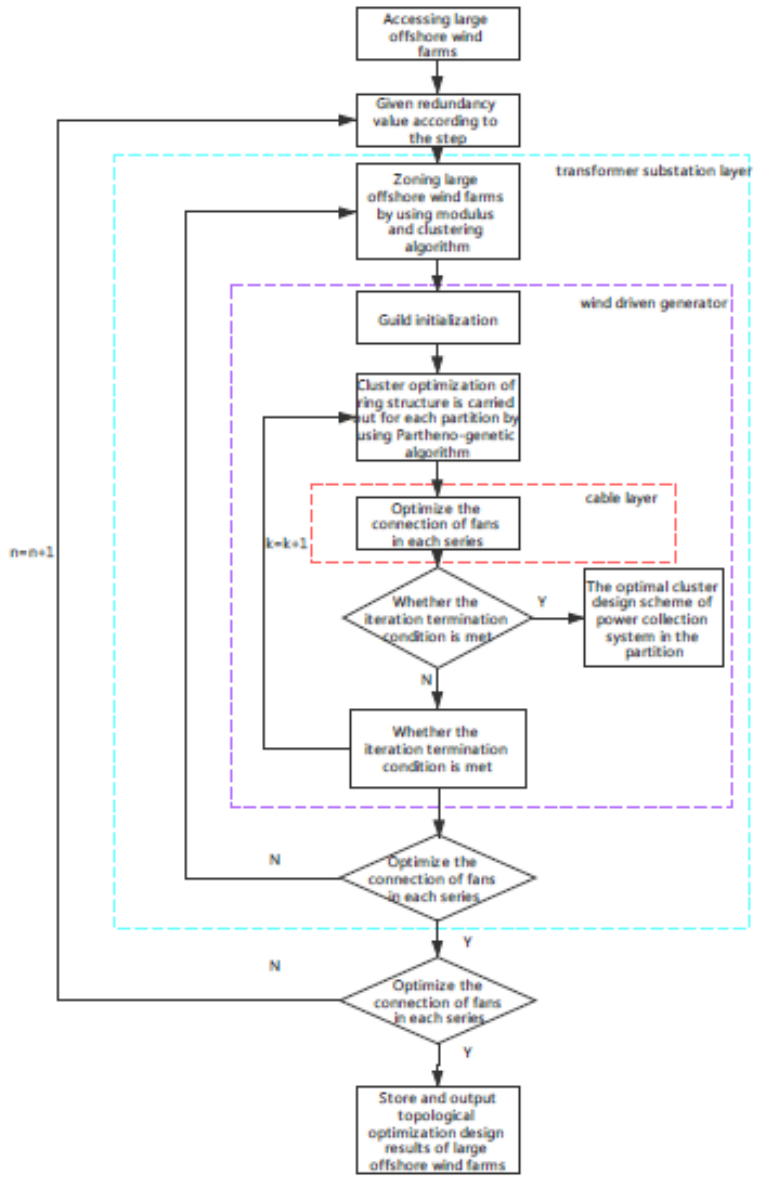

Fig. 2. Flow chart of topology redundancy optimization design of power collector system.

\section{Case Analysis}

\subsection{Case Description}

The case is A large-scale offshore wind farm with A total capacity of 100 single wind turbines of $3.6 \mathrm{MW}$, which is $38 \mathrm{~km}$ offshore. The specific parameters of the offshore wind turbines are shown in Table A1 in Appendix A. The coordinates of the fans in the offshore wind farm have been determined, and the arrangement is shown in Figure. A1 in Appendix A. The connection between fans in the collector system adopts $35 \mathrm{kV}$ sea cable, and the transmission cable adopts $220 \mathrm{kV}$ high voltage sea cable. The main parameters of $35 \mathrm{kV}$ sea cables are provided by the design institute, as shown in Table A2 of Appendix A. According to the bearing capacity and short-circuit characteristics of the submarine cable, the current of the fan with the capacity of $3.6 \mathrm{MW}$ through the $35 \mathrm{kV}$ medium-voltage Marine cable is $3.6 \mathrm{MW} /(1.732 \times 35 \mathrm{kV}) \approx 59 \mathrm{~A}$. The power collector circuit adopts a radial structure, and the cable section is determined by the number of fans in the connecting series. If the annular structure is adopted, the cable section should meet the "N-1" principle, which means the selected cable section should meet the fault at any point in the ring, and the cable can satisfy the load flow required for normal power generation under the operating condition of the fan. The current carrying capacity of the $500 \mathrm{~mm} 2$ MEDIUM-voltage cable is $630 \mathrm{~A}$. Since $630 \mathrm{~A} / 59 \mathrm{~A} \approx 10$, the maximum current flow of the $500 \mathrm{~mm} 2$ medium-voltage cable is the sum of 10 fans with a capacity of $3.6 \mathrm{MW}$. If the quantity exceeds it, there will be no thicker cable to match it. As a result, the cable selection cannot be carried out.

Parameters such as the number of failures and repair time of related equipment could be seen in literature [9].

\subsection{Case simulation results}

Implement topology optimization for different redundancy power collector system, then do optimization for partition of wind farms to achieve two optimal division. The simulation result shows topology optimization design of the structure when the redundancy was $0.3822,0.41313,0.43243$. As is shown in figure 3 , including an asterisk fan, black circles represent booster station at sea.

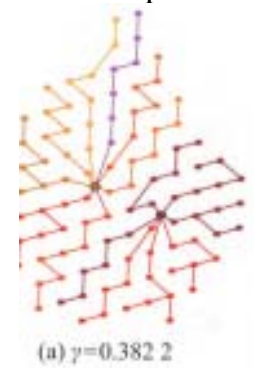

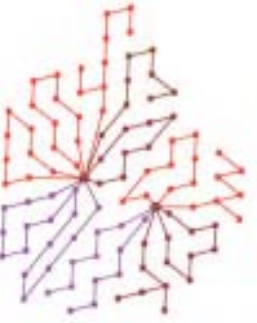

(b) $\gamma=0.413 \quad 13$

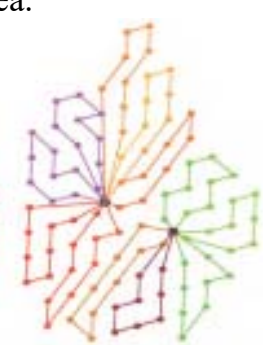

(c) $y=0.43243$
Fig. 3. Structures with topology optimization under different redundancies

\subsection{Game of economy and reliability under different redundancy}

The redundancy design of large offshore wind farm can improve its reliability, but the redundancy design also increases its economic cost. Meanwhile the economy and reliability of the power collector system with different redundancy design are different. Therefore, the economic cost and reliability cost of different redundancy design of the collector system should be analyzed concretely, and the advantages and disadvantages of different redundancy design should be compared as well.

Figure. 4 shows the curve of initial investment cost, operation and maintenance cost, power failure loss cost and network loss cost of the collector system under different redundancies, as well as the relationship between the full life cycle cost of the collector system and redundancies. 

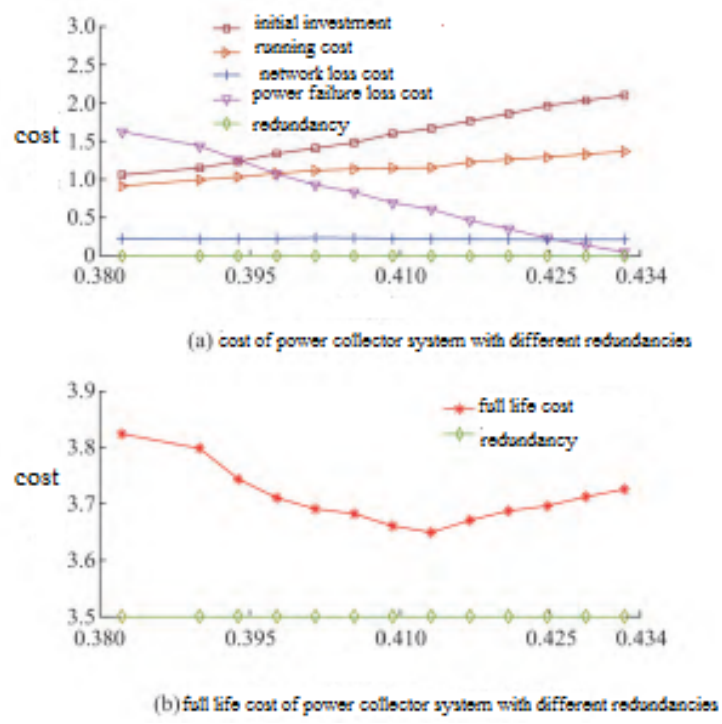

Fig. 4. Cost curves of optimized topology for power collector system with different redundancies.

It can be seen from Figure. 4 (a) that the initial investment cost and operation and maintenance cost of the power collector system rise with the increase of redundancy, and the initial investment cost of the collector system increases faster under different redundancy. When power collector system redundancy is 0.3822 , topology optimization of the structure is simple, the number of submarine cable and the switch devices is small. So the initial investment cost and operational cost is minimum. However, as redundancy, medium-voltage cable and the number of switch equipment increases, initial cost grows and increased runtime failure frequency leads to more operational cost.

When the redundancy of the collector system is 0.43243 , the initial investment cost with operation and maintenance cost of the topological optimization structure is the maximum. The initial investment cost is approximately twice of that when the redundancy is 0.3822 .

From the perspective of the full life cycle of the offshore wind farm, the power loss caused by the failure of the medium-voltage sea cable or switch equipment is also an important part of the total cost of topology redundancy design of the power collector system. It can be seen from Figure. 4 (a) that the failure loss cost of the collector system decreases with the increase of the redundancy of the optimized structure, while the network loss cost of the collector system has little difference among the design schemes with different redundancy levels. When the redundancy of the power collector system is 0.43243 , the power loss cost of the topological optimization structure is the lowest, which is only $3.05 \%$ of that of the topological optimization structure with the highest power loss cost (redundancy is 0.3822 ). By the way, the cost of power failure is less than the cost of network loss.

It can be seen from Figure. 4 (b) that, from the perspective of the full life cycle of the wind farm power collector system, the total cost of the topological optimization structure when the redundancy of the collector system is 0.43243 is lower than that of the topological optimization structure when the redundancy is 0.3822 , which has certain economic advantages. Compared the full life cycle of topological optimization structure with different redundancy of the power collector system, the cost is minimum when redundancy is 0.41313 . As a result, it is the optimal structural design for the full life cycle of the power collector system. As shown in Figure. 3 (b), this scheme is referred to as the optimal redundancy scheme for short.

Figure 5 gives the full life cycle cost curve of the optimal radiation shape scheme, the optimal redundancy scheme and the ring optimal scheme, as shown in Figure 5.

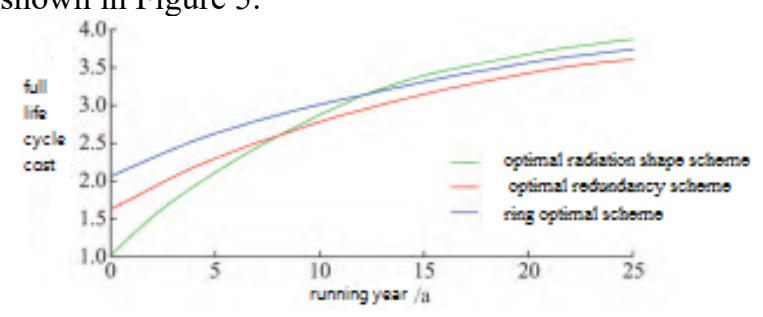

Fig. 5. Life cycle cost curves for three typical structures with optimal topologies.

As can be seen from Figure 5, among the optimal connection methods under the three typical structures, the initial investment cost is sorted as: ring optimal scheme $>$ optimal scheme of redundancy $>$ radial optimal scheme. Ring optimal scheme and optimal scheme of redundancy have higher reliability at the cost of a certain economic cost. After running up to 8 years, the optimal scheme of redundancy begins to be better than the radial structure. After running up to 12 years, the full life cycle cost of the ring structure began to be better than the radial structure. By the 25th year, the full life cycle cost of the three typical structures was sorted as: radial optimal scheme>ring optimal scheme> optimal scheme of redundancy. For the topological structure with optimal redundancy, the full life cycle cost has obvious advantages over the other two classical structures, while the initial investment cost is higher than the radial structure

\section{Conclusion}

Based on the characteristics of the typical radial and ring topology of the offshore wind farm power collector system, this paper proposes a topological structure with partial redundancy, establishes a total cost optimization model for the full life cycle of the redundancy of the power collector system, and gives a specific optimization flow chart. By analyzing a planned case of large offshore wind farms, the following conclusions can be drawn.

1) The power collector system adopts the topological structure of partial redundancy. The economy and reliability are between the radial and the full ring topology structures. From the view of full life 
cycle cost of the power collector system, the optimal power collector system topological structure has high reliability and certain economic advantages.

2) Under the existing conditions, the full ring structure has economic advantages over the radial structure after about 12 years' operation of the wind farm, and the structure with optimal redundancy has obvious economic advantages compared to the radial structure after the wind farm operation for about 8 years.

In this paper, the capacity of wind turbines selected by offshore wind farms is $3.6 \mathrm{MW}$, wind turbines of large-scale far-reaching offshore wind farm capacity will reach $6 \mathrm{MW}, 8 \mathrm{MW}$, or even $10 \mathrm{MW}$. Wind turbines exert a great impact on the optimization of centralized system planning as the single capacity becomes larger. In the next stage, analysis about optimization of the design criteria of the power collector system, substation type, medium voltage cable level will be combined with the characteristics of the far-reaching offshore wind farm high-capacity wind turbines.

\section{References}

1. Wang Xifan, Wang Biyang, Wang Xiuli, et al. Automation of Electric Power Systems, 38(17), 413(2014)

2. Quinonez-Varela G, Ault G W, Anaya-Lara O, et al. IET Renewable Power Generation, 1(2), 107114(2007)

3. Jiang Nan, New Energy Progress, 3(1), 21-24(2015)

4. Chen Ying, Dong Zhaoyang, Meng $\mathrm{Ke}$, et al. Journal of Modern Power Systems and Clean Energy, 1(3), 258-263(2013).

5. Chen Ning, Research on optimization of power collector system for large-scale offshore wind farms, Shanghai University of Electric power(2011)

6. Fu Yang, Wu Jing, Wei Shurong, et al. Topology optimization and planning of power collection system for large scale offshore wind farm. Power System Technology, 37(9), 2553-2558(2013)

7. Wang Jiandong, Li Guojie, Economic comparison of different collector networks for offshore wind farm, Automation of Electric Power Systems, 33(11), 99-103(2009)

8. Wang Jiandong, Li Guojie, Economic study on switchgear configuration in electrical systems of offshore wind farms with cable outage, Power System Technology, 34(2), 125-128(2010)

9. Wei Shurong, Zhang $\mathrm{Lu}, \mathrm{Xu}$ Yao, et al. Hierarchical optimization for the double-sided ring structure of the collector system planning of large offshore wind farms. IEEE Transactions on Sustainbale Energy, 8(3), 1029-1039(2017)

10. $\mathrm{Fu}$ Yang, $\mathrm{Xu}$ Hanlu, Huang Lingling, Offshore wind farm collector system of life cycle cost analysis. Automation of Electric Power Systems, 40(21), 161-167(2016)
11. Yang Jin, O’Reilly J, Fletcher J E, Redundancy analysis of offshore wind farm collection and transmission systems, International Conference on Sustainable Power Generation and Supply, April 67, 7(2009)

12. Yang Jin, O'Reilly J, Fletcher J E, Reliability enhancement of offshore wind farms by redundancy analysis, Auyomation of Electric Power System, 34(4), 84-91(2010)

13. Sun Junyang, Zhu Miao, Gao Qiang, et al, Optimal design of internal topology for high power AC offshore wind farm, Power System Technology, 37(7), 1978-1982(2013)

14. Tan Renshen, Yang Ping, He Peng, et al, Study on reliability and sensitivity of offshore wind farm collector system considering electrical fault and switch configuration scheme, Power System Technology, 37(8), 2264-2270(2013)

15. Scutariu M. Techno-economical optioneering of offshore wind farms electrical systems, IEEE lausanne Power Tech, July 1-5, Lausanne, Switzeland, 2195-2200(2007)

16. Zhang Jianping, Zhu Zhongjie, Liu lu, et al. Transmission network value planning considering large-scale wind power integration, Automation of Electric Power Systems, 38(19), 47-51(2014)

17. Su Haifeng, Zhang Jianhua, Liang Zhirui, et al, Substation Locating and Sizing optimization planning based on life cycle cost, Automation of Electric Power Systems, 36(23), 59-64(2012)

18. Su Haifeng, Zhang Jianhua, Liang Zhirui, et al, Substation LCC Planning based on refined mean clustering random particle swarm algorithm, Transactions of China Electrotechnical Society, 27(4), 209-215(2012) 